\title{
Dysbiosis in food allergy and implications for microbial therapeutics
}

\section{Cecilia Berin}

Jaffe Food Allergy Institute, Icahn School of Medicine at Mount Sinai, New York, New York, USA

\begin{abstract}
The increase in food allergy prevalence in recent years suggests that environmental factors, such as diet and intestinal microbiota, play contributory roles. In this issue of the $J C I$, Bao et al. compared twins that differed with respect to food allergies. The researchers analyzed sequences from microbe ribosomal RNA and profiled microbe metabolites, identifying health-associated microbes at the species level. In addition to revealing microbes from the Clostridia class enriched in healthy twins, the authors identified two commensal species (Phascolarctobacterium faecium and Ruminococcus bromii) related to the healthy fecal metabolome. This study advances the goal for next-generation probiotic therapies that effectively treat or prevent food allergy.
\end{abstract}

cy between studies. Factors such as age, diet, genetic background, and environmental exposure influence the microbiota and make it difficult to delineate patterns associated with disease between distinct and relatively small cohorts.

The most common technique to survey microbial communities relies on ribosomal gene (16S rRNA) analysis. 16S rRNA sequencing is typically limited to taxonomic classification at the family or genus level because only short $16 \mathrm{~S}$ rRNA gene regions are sequenced. Furthermore, the overlapping effect of different organisms on host immunity (for example, the ability of different strains to generate immunomodulatory metabolites, such as short-chain fatty acids) make it difficult to identify functional differences in the microbiota using low-resolution genetic tools.

allergy in the United States increased from $0.2 \%$ to $1.1 \%$ between the years of 1997 and 2008 , and peanut allergy increased from $0.4 \%$ to $1.4 \%$ in the same time period (1). This remarkable increase in the number of people with allergies is the focus of intense scrutiny, as such a rapid increase suggests the influence of an important environmental factor, such as diet. Until 2008, the American Academy of Pediatrics recommended delaying the introduction of allergenic foods into the infant diet in those with a family history of allergic disease. Due to poorly supporting clinical data, the recommendations for avoidance were removed in 2008. In 2015, the LEAP study found that dietary exposure to peanut beginning between 5 and 11 months of age profoundly suppressed the development of peanut allergy (2). Infant feeding guidelines were subsequently modified to encourage early introduction of peanut into the diet (3).

In addition to diet, other important parameters associate with elevated or reduced risk for development of food allergy. Interestingly, exposure to a dog in the home in the first year of life consistently associates with protection from food allergy (4) and other allergic diseases (5). Mechanistic studies of the relationship between early life dog exposure and reduced asthma risk link protection to changes in the intestinal microbiota (6). Several studies have demonstrated that individuals with established food allergy have dysbiosis (7-12), while other studies have identified infant dysbiosis preceding the development of food allergy $(13,14)$. A recent study associated maternal Prevotella colonization with protection from food allergy in offspring, independent of infant Prevotella colonization (15). Dysbiosis in established disease may be due to intestinal inflammation or changes in diet (i.e., a consequence of disease rather than a causative factor), while dysbiosis preceding disease is consistent with, but not proof of, a contributing role in pathogenesis. While many studies identify dysbiosis in food allergy, the specific microbial populations altered in food allergy lack clear consisten-

\section{Related Article: https://doi.org/10.1172/JCl141935}

Conflict of interest: The author has declared that no conflict of interest exists.

\section{Twins discordant for food allergy}

In this issue of the JCI, Bao et al. (16) use a powerful twin study design to examine how fecal microbiota and metabolites differ between twins discordant for food allergy. Twins share their early life environment, which is a critical window for developing microbiota, the immune system, and food allergies. Moreover, the shared genetic background of monozygotic twins eliminates many confounding factors from the study of diseaseassociated differences in microbiota. The researchers analyzed 18 twin pairs ranging in age from 0.5 to 58 years, including 13 disease-discordant twin pairs and 5 twin pairs who were both affected with food allergy. They analyzed 16S rRNA amplicon sequences between twin pairs and identified 64 differentially operational taxonomic units (OTUs), 62 more abundant in healthy twins and 2 more abundant in allergic twins. Most healthy-abundant OTUs were in the Clostridia class and were annotated as Lachnospiraceae and Ruminococcaceae. Interestingly, Lachnospiraceae associates with protection 
from milk allergy in mice colonized with healthy human microbiota (17).

Bao and colleagues also performed fecal metabolomics and identified 97 metabolites that were differentially abundant between healthy and allergic twins (16). Although short-chain fatty acids are microbial-derived metabolites that may promote tolerance, no differences were observed between the groups. In contrast, the diacylglycerol pathway was notably enriched in healthy twins. The investigators then analyzed the correlation between differentially abundant OTUs and differentially abundant metabolites. They matched two of the metabolome-correlating OTUs to the species level, Phascolarctobacterium faecium and Ruminococcus bromii, and showed an increased abundance in healthy twins. $P$. faecium was highly correlated with diacylglycerol metabolites, while $R$. bromii, which is known to play a role in starch digestion, was associated with metabolites involved in fatty acid, amino acid, and sterol metabolism. This study uses a unique twin design and strategically combines genetic and metabolomic analysis to identify microbes and their products associated with food allergy (16).

\section{The functional consequence of food allergy-associated dysbiosis}

The key next step will be to determine the functional consequences of colonization with organisms identified by Bao et al. (16) Previous studies have determined the functional consequence of food allergyassociated dysbiosis by transferring human microbiota into germ-free or antibiotictreated mice $(12,17)$. When researchers transferred microbiota from healthy donors, but not allergic donors, food allergy was prevented $(12,17)$. Notably, healthy microbiota averted food allergy by improving intestinal barrier function $(17,18)$ and expanding ROR $\mathrm{t}^{+}$- and Foxp $3^{+}$-expressing Tregs (12). Similar to Bao et al. (16), previous studies, which administered bacteria from healthy donors as an oral inoculation, also identified protective species within the Clostridia class $(12,16)$. Other studies have focused on the functional consequence of metabolic changes. Dysbiosis in infants with a high risk for multisensitized allergic disease was associated with elevated levels of 12,13-diHOME, a lipid fatty acid capable of driving Th2 sensitization in human immune cells in vitro (19). Furthermore, administering this lipid metabolite to mice could exacerbate experimental asthma by suppressing Tregs (20).

\section{Clinical implications}

The demonstration that microbes determine susceptibility to food allergy in experimental systems has led to a great deal of excitement about the possibility of microbial-based therapeutics. Fecal microbial transplants, involving the transplant of stool-derived microbiota from a healthy individual to an allergic recipient, are currently underway (ClinicalTrials.gov NCT02960074). However, such approaches are crude and carry risk (21). The administration of the probiotic Lactobacillus rhamnosus together with peanut oral immunotherapy (OIT) protected subjects from (22) peanut allergy, and another clinical trial is examining whether the combination of peanut OIT plus L. rhamnosus is more effective than OIT alone (ACTRN12616000322437). A clinical trial of a selected consortium (VE416) of human commensal clonal strains that suppress allergic disease is currently underway for the treatment of peanut allergy (ClinicalTrials.gov NCT03936998). However, the promise brought by human dysbiosis research is held in the identity of protective microbes or their metabolites. Rational selection of a human commensalbased therapeutic that has the capacity to colonize the human gastrointestinal tract could effectively prevent food allergy. For clinicians to move from studying human dysbiosis to providing therapy, it is necessary to identify protective organisms, ideally at the strain level; to identify metabolites associated with tolerance; and to demonstrate the corresponding regulatory functions in appropriate model systems. There is also a question of whether there is a critical window of opportunity for microbes to regulate $\operatorname{IgE}$ in early life, as has been suggested by mouse studies (23). Bao et al. (16) demonstrated that subjects with established food allergy also had dysbiosis; however, it is unclear if normalizing the microbiota would suppress entrenched Th2 and IgE responses. In addition, it is unknown whether treatments with commensal organisms would require cotreatment with the allergen itself in order to reestablish antigen-specific oral tolerance. Further, maintaining healthy microbiota in an allergic host may require additional factors, such as a specialized diet.

In summary, human food allergy associates with dysbiosis, which has consequences for the development of tolerance or allergy, as shown experimentally in mouse models. With the latest addition to the field by Bao et al. (16), two additional human commensal species ( $P$. faecium and $R$. bromii) relate to the healthy fecal metabolome, bringing us a step closer to treating food allergies with next-generation probiotics.

Address correspondence to: M. Cecilia Berin, Jaffe Food Allergy Institute, 1425 Madison Ave., 11-23A, Icahn School of Medicine at Mount Sinai, New York, New York 10029, USA. Phone: 212.659.1493; Email: cecilia.berin@mssm.edu.

1. Sicherer SH, et al. US prevalence of selfreported peanut, tree nut, and sesame allergy: 11-year follow-up. J Allergy Clin Immunol. 2010;125(6):1322-1326.

2. Du Toit G, et al. Randomized trial of peanut consumption in infants at risk for peanut allergy. N Engl J Med. 2015;372(9):803-813.

3. Togias A, et al. Addendum guidelines for the prevention of peanut allergy in the United States: summary of the National Institute of Allergy and Infectious Diseases-sponsored expert panel. J Acad Nutr Diet. 2017;117(5):788-793.

4. Peters RL, et al. Differential factors associated with challenge-proven food allergy phenotypes in a population cohort of infants: a latent class analysis. Clin Exp Allergy. 2015;45(5):953-963.

5. Ownby DR, et al. Exposure to dogs and cats in the first year of life and risk of allergic sensitization at 6 to 7 years of age. JAMA. 2002;288(8):963-972.

6. Fujimura KE, et al. House dust exposure mediates gut microbiome Lactobacillus enrichment and airway immune defense against allergens and virus infection. Proc Natl Acad Sci U S A. 2014;111(2):805-810.

7. Hua X, et al. Allergy associations with the adult fecal microbiota: analysis of the American Gut Project. EBioMedicine. 2016;3:172-179.

8. Ling Z, et al. Altered fecal microbiota composition associated with food allergy in infants. Appl Environ Microbiol. 2014;80(8):2546-2554.

9. Goldberg MR, et al. Microbial signature in IgE-mediated food allergies. Genome Med. 2020;12(1):92.

10. Bunyavanich S, et al. Early-life gut microbiome composition and milk allergy resolution. J Allergy Clin Immunol. 2016;138(4):1122-1130.

11. Fazlollahi M, et al. Early-life gut microbiome and egg allergy. Allergy. 2018;73(7):1515-1524.

12. Abdel-Gadir A, et al. Microbiota therapy acts via a regulatory $\mathrm{T}$ cell MyD88/RORgammat path- 
way to suppress food allergy. Nature medicine. 2019;25(7):1164-1174.

13. Azad MB, et al. Infant gut microbiota and food sensitization: associations in the first year of life. Clin Exp Allergy. 2015;45(3):632-643.

14. Savage JH, et al. A prospective microbiome-wide association study of food sensitization and food allergy in early childhood. Allergy. 2018;73(1):145-152.

15. Vuillermin PJ, et al. Maternal carriage of Prevotella during pregnancy associates with protection against food allergy in the offspring. Nat Commun. 2020;11(1):1452.

16. Bao R, et al. Fecal microbiome and metabolome differ in healthy and food-allergic twins. J Clin Invest. 2020;131(2):e141935.

17. Feehley T, et al. Healthy infants harbor intestinal bacteria that protect against food allergy. Nat Med. 2019;25(3):448-453.

18. Stefka AT, et al. Commensal bacteria protect against food allergen sensitization. Proc Natl Acad Sci U S A. 2014;111(36):13145-13150.

19. Fujimura KE, et al. Neonatal gut microbiota associates with childhood multisensitized atopy and T cell differentiation. Nat Med. 2016;22(10):1187-1191.

20. Levan SR, et al. Elevated faecal 12,13-diHOME concentration in neonates at high risk for asthma is produced by gut bacteria and impedes immune tolerance. Nat Microbiol. 2019;4(11):1851-1861.

21. Wang S, et al. Systematic review: adverse events of fecal microbiota transplantation. PLoS One. 2016;11(8):e0161174.

22. Tang ML, et al. Administration of a probiotic with peanut oral immunotherapy: A randomized trial. J Allergy Clin Immunol. 2015;135(3):737-744.

23. Cahenzli J, et al. Intestinal microbial diversity during early-life colonization shapes long-term IgE levels. Cell Host Microbe. 2013;14(5):559-570. 